\title{
Investigations on the Relativistic Interactions in One-Electron Atoms with Modified Yukawa Potential for Spin 1/2 Particles
}

\author{
Abdelmadjid Maireche ${ }^{1, a^{*}}$ \\ ${ }^{1}$ Laboratory of Physics and Material Chemistry, Physics Department, Sciences Faculty, \\ University of M'sila-M'sila Algeria \\ *a abmaireche@gmail.com
}

\begin{abstract}
Keywords: Yukawa potential, noncommutative space, star product, Bopp's shift method and Dirac equation.
\end{abstract}

\begin{abstract}
Energy levels of one electron atoms have been re-examined by applying an alternative perturbative scheme in solving the modified Dirac equation (m.d.e.) for the modified Yukawa potential model with an arbitrary spin-orbit quantum number $(k=+l,-(l+1), \tilde{k}=+\tilde{l},-(\tilde{l}+1))$ by means Bopp's shift method instead to solving (m.d.e.) with star product, in the framework of noncommutativity three dimensional real space (NC: 3D-RS). It is observed that the obtained corrections of energies are depended on the new discrete atomic quantum numbers $(k, \tilde{k}, m, \tilde{m})$ under spin-symmetry and pseudospin symmetry and two infinitesimal parameters $\left(\Theta_{i j}, \chi_{i j}\right) \equiv \varepsilon_{i j}^{k}\left(\Theta_{k}, \chi_{k}\right)$ which induced by position-position noncommutativity. Furthermore, in limit of parameters $\left(\Theta_{k}, \chi_{k}\right) \rightarrow(0,0)$, the new energy equations for modified Yukawa potential are consistent with the results of ordinary relativistic quantum mechanics for ordinary Yukawa potential.
\end{abstract}

\section{Introduction}

In very recent years the energy levels of atoms, nuclei, and hadrons have been studied by several analytic methods in the case the two relativistic equations Klein-Gordon and Dirac for certain potentials of the physical interest [1-21], some of these potentials are known to play important roles in many fields, one of such potential is the Yukawa potential has been a subject of many studies, it is a central potential of nuclear shell model in addition to used in nonrelativistic quantum mechanics to study the interactions between nonrelativistic particles, in atomic and molecular physics, it represents a screened Coulomb potential due to the cloud of electronic charges around the nucleus, on the other hand, it has also been used to describe the interaction between charged particles in plasma, solid and colloidal suspensions [22]. It is well known that the ordinary quantum structures obey the standard Weyl-Heisenberg algebra in both Schrödinger and Heisenberg (the operators are depended on time) pictures ((HP) and (SP)), respectively, as (Throughout this paper the natural unit $c=\hbar=1$ are employed):

$$
\begin{aligned}
& \left\lfloor x_{i}, p_{j}\right\rfloor=\left\lfloor x_{i}(t), p_{j}(t)\right\rfloor=i \delta_{i j} \\
& {\left[x_{i}, x_{j}\right]=\left[p_{i}, p_{j}\right]=\left[x_{i}(t), x_{j}(t)\right]=\left[p_{i}(t), p_{j}(t)\right]=0}
\end{aligned}
$$

where the operators $\left(x_{i}(t), p_{i}(t)\right)$ in (HP)are related to the corresponding operators $\left(x_{i}, p_{i}\right)$ in (SP) from the following projections relations:

$$
\left(x_{i}, p_{i}\right) \Rightarrow\left(x_{i}(t), p_{i}(t)\right)=\exp \left(i \hat{H}_{c o m-y p}\left(t-t_{0}\right)\right)\left(x_{i}, p_{i}\right) \exp \left(-i \hat{H}_{c o m-y p}\left(t-t_{0}\right)\right)
$$

here $\hat{H}_{c o m-y p}$ denote to the ordinary quantum Hamiltonian operator for Yukawa potential. In addition, for spin $1 / 2$ particles described by the Dirac equation, experiment tells us that must satisfy Fermi Dirac statistics obey the restriction of Pauli, which imply to gives the only non-null equaltime anti-commutator for field operators as follows: 


$$
\left\{\Psi_{\alpha}(t, \mathrm{r}), \bar{\Psi}_{\beta}\left(t, \mathrm{r}^{\prime}\right)\right\}=i\left(\gamma^{0}\right)_{\alpha \beta} \delta^{3}\left(\mathrm{r}-\mathrm{r}^{\prime}\right)
$$

Very recently, many authors have worked on solving the fundamental equations (Schrödinger, Klein-Gordon and Dirac equations) with physical potential in the new structure of quantum mechanics, known by NC quantum mechanics which known firstly by H. Snyder, it is shown a direct relationship between string theory and noncommutative geometry and to obtaining profound and new applications for different areas of matter sciences in the microscopic and nano scales within the framework of quantum mechanics and quantum field theory [23-71]. It is important to noticing that, the new quantum structure of $\mathrm{NC}$ space based on the following $\mathrm{NC}$ canonical commutations relations (NCCRs) in both (HP) and (SP)), as follows [24-60]:

$$
\left[\hat{x}_{i}^{*}, \hat{p}_{j}\right]=\left[\hat{x}_{i}(t)^{*}, \hat{p}_{j}(t)\right]=i \delta_{i j},\left[\hat{x}_{i}^{*}, \hat{x}_{j}\right]=\left[\hat{x}_{i}(t)^{*}, \hat{x}_{j}(t)\right]=i \theta_{i j} \text { and }\left[\hat{p}_{i}^{*}, \hat{p}_{j}\right]=\left[\hat{p}_{i}(t)^{*}, \hat{p}_{j}(t)\right]=0
$$

the new operators $\left(\hat{x}_{i}(t), \hat{p}_{i}(t)\right)$ in (HP) are related to the corresponding new operators $\left(\hat{x}_{i}, \hat{p}_{i}\right)$ in (SP) from the new projections relations [27-33]:

$$
\left(\hat{x}_{i}, \hat{p}_{i}\right) \Rightarrow\left(\hat{x}_{i}(t), \hat{p}_{i}(t)\right)=\exp \left(i \hat{H}_{n c-y p}\left(t-t_{0}\right)\right) *\left(\hat{x}_{i}, \hat{p}_{i}\right) * \exp \left(-i \hat{H}_{n c-y p}\left(t-t_{0}\right)\right)
$$

with $\hat{H}_{n c-y p}$ being the Hamiltonian operator of the extended quantum system described on (NC: 3DRS) symmetries. The very small parameters $\theta^{\mu v}$ (compared to the energy) are elements of antisymmetric real matrix of dimension $\frac{(\text { length })^{2}}{\hbar}$ and $(*)$ denote to the new star product (the MoyalWeyl product), which is generalized between two arbitrary functions $f(x) \rightarrow \hat{f}(\hat{x})$ and $g(x) \rightarrow \hat{g}(\hat{x})$ to $\hat{f}(\hat{x}) \hat{g}(\hat{x}) \equiv(f * g)(x)$ instead of the usual product $(f g)(x)$ in ordinary three dimensional spaces [23-71]:

$$
(f g)(x, p) \Rightarrow \hat{f}(\hat{x}) \hat{g}(\hat{x}) \equiv(f * g)(x)\left(f g-\left.\frac{i}{2} \theta^{\mu \nu} \partial_{\mu}^{x} f \partial_{\nu}^{x} g\right|_{\left(x^{\mu}=x^{\nu^{\nu}}\right)}+O\left(\theta^{2}\right)\right.
$$

where $\hat{f}(\hat{x})$ and $\hat{g}(\hat{x})$ are the new function in (NC: 3D-RS), the following term $\left(-\frac{i}{2} \theta^{\mu v} \partial_{\mu}^{x} f(x) \partial_{v}^{x} g(x)\right)$ is induced by (space-space) noncommutativity properties and $O\left(\theta^{2}\right)$ stands for the second and higher order terms of $\theta$, a Bopp's shift method can be used, instead of solving any quantum systems by using directly star product procedure [23-71]:

$$
\left\lfloor\hat{x}_{i}, \hat{x}_{j}\right\rfloor=\left\lfloor\hat{x}_{i}(t), \hat{x}_{j}(t)\right\rfloor=i \theta_{i j} \text { and }\left\lfloor\hat{p}_{i}, \hat{p}_{j}\right\rfloor=\left\lfloor\hat{p}_{i}(t), \hat{p}_{j}(t)\right\rfloor=0
$$

The three-generalized coordinates $\left(\hat{x}=\hat{x}_{1}, \hat{y}=\hat{x}_{2}, \hat{z}=\hat{x}_{3}\right)$ in the $\mathrm{NC}$ space are depended with corresponding three-usual generalized positions $(x, y, z)$ and three momentums coordinates $\left(p_{x}, p_{y}, p_{z}\right)$ by the following relations, as follows [28-43]:

$$
\hat{x}_{i}=x_{i}-\frac{1}{2} \theta_{i j} p_{j} \Rightarrow\left\{\begin{array}{l}
\hat{x}=x-\frac{\theta_{12}}{2} p_{y}-\frac{\theta_{13}}{2} p_{z}, \\
\hat{y}=y-\frac{\theta_{21}}{2} p_{x}-\frac{\theta_{23}}{2} p_{z}, \\
\hat{z}=z-\frac{\theta_{31}}{2} p_{x}-\frac{\theta_{32}}{2} p_{y} .
\end{array}\right.
$$


The non-vanish-commutators in (NC-3D: RS) can be determined as follows:

$$
\begin{aligned}
& {\left[\hat{x}, \hat{p}_{x}\right]=\left\lfloor\hat{y}, \hat{p}_{y}\right\rfloor=\left[\hat{z}, \hat{p}_{z}\right]=i,} \\
& {[\hat{x}, \hat{y}]=i \theta_{12},[\hat{x}, \hat{z}]=i \theta_{13},[\hat{y}, \hat{z}]=i \theta_{23}}
\end{aligned}
$$

which allow us to getting the operator $\hat{r}^{2}$ on NC three dimensional spaces as follows [28-34]:

$$
\hat{r}^{2}=r^{2}-\overrightarrow{\mathbf{L}} \vec{\Theta}
$$

the coupling $\mathrm{L} \Theta \equiv L_{x} \Theta_{12}+L_{y} \Theta_{23}+L_{z} \Theta_{13}$ with $\Theta_{i j}=\theta_{i j} / 2, L_{x}=y p_{z}-z p_{y}, L_{y}=\mathrm{zp}_{\mathrm{x}}-\mathrm{xp}_{\mathrm{z}}$ and $L_{z}=x p_{y}-y p_{x}$. Furthermore, the new equal-time anti-commutator for fermionic field operators' in NC spaces can be expressed as [28-34]:

$$
\begin{aligned}
& \left\{\hat{\Psi}_{\alpha}(t, \mathrm{r}), \hat{\Psi}_{\beta}\left(t, \mathrm{r}^{\prime}\right)\right\}=i\left(\gamma^{0}\right)_{\alpha \beta} \delta^{3}\left(\mathrm{r}-\mathrm{r}^{\prime}\right) \\
& \left\{\hat{\Psi}_{\alpha}(t, \mathrm{r}), \hat{\Psi}_{\alpha}\left(t, \mathrm{r}^{\prime}\right)\right\}=\left\{\hat{\bar{\Psi}}_{\alpha}(t, \mathrm{r}), \hat{\bar{\Psi}}_{\beta}\left(t, \mathrm{r}^{\prime}\right)\right\}=i \theta_{\alpha \beta} \delta^{3}\left(\mathrm{r}-\mathrm{r}^{\prime}\right)
\end{aligned}
$$

The purpose of the present work is to extend and present the solution of the Dirac equation with spin-1/2 particle moving in modified Yukawa potential of the new form:

$$
V_{m y}(\hat{r})=v_{0} \frac{e^{-\alpha r}}{r}+\left\{\begin{array}{l}
v_{0}(1+\alpha) \frac{\vec{L} \vec{\Theta}}{2 r^{3}} e^{-\alpha r} \overrightarrow{\mathbf{L} \Theta} \text { for the spin symmetric case } \\
v_{0}(1+\alpha) \frac{\vec{L} \vec{\Theta}}{2 r^{3}} e^{-\alpha r} \widetilde{\overrightarrow{\mathbf{L}}} \vec{\Theta} \text { for thep - spin symmetric case }
\end{array}\right.
$$

In (NC: 3D-RS) using the generalization Bopp's shift method to discover the new symmetries and a possibility to obtain another applications to this potential in different fields. This work based essentially on our previously works [23-50]. The plan of recently work is as follows: In next section, we briefly review the Dirac equation with Yukawa potential on based to refs. [18-22]. In section three, we give a description of the Bopp's shift method for (m.d.e.) with modified Yukawa potential. Then in section four, we apply standard perturbation theory to establish exact modifications at first order of infinitesimal parameters $(\Theta, \chi)$ for the perturbed Dirac equation in (NC-3D: RS) for spin-orbital (pseudo-spin orbital) and the relativistic magnetic spectrum for modified Yukawa potential. Section fifth is devoted for discussion the global spectrum and corresponding NC Hamiltonian for modified Yukawa potential. Finally, we give a brief conclusion in last section.

\section{Review of the Dirac equation for Yukawa potential in relativistic quantum mechanics}

Let us start this section by the review of a relativistic particle in spherically symmetric for the potential $V(r, \theta)$ which known by Yukawa potential on based to the main reference [22]:

$$
V(r)=v_{0} \frac{e^{-\alpha r}}{r}
$$

where $\alpha$ and $v_{0}$ denote to the rang of nuclear force and strength of potential, respectively. The Dirac equation describing a fermionic particle (spin-1/2 particle) with scalar $S(r, \theta)$ and vector $V(r, \theta)$ potentials is given by [18-22]:

$$
(\alpha \mathrm{P}+\beta(M+S(r, \theta))) \Psi(r, \theta, \varphi)=(E-V(r, \theta)) \Psi(r, \theta, \varphi)
$$


here $M$ are $E$ the fermions' mass and the relativistic energy while

$$
\left(\alpha_{i}=\left(\begin{array}{cc}
0 & \sigma_{i} \\
\sigma_{i} & 0
\end{array}\right), \beta=\left(\begin{array}{cc}
I_{2 \times 2} & 0 \\
0 & I_{2 \times 2}
\end{array}\right)\right)
$$

are the usual Dirac matrices, the spinor $\Psi(r, \theta, \varphi)$ can be expressed as [18-22]:

$$
\left.\Psi_{n k}(r, \theta, \varphi)=\left(\begin{array}{l}
f_{n k}(\vec{r}) \\
g_{n k} \\
\vec{r}
\end{array}\right)\right)=\frac{1}{r}\left(\begin{array}{l}
F_{n k}(r) Y_{j m}^{l}(\theta, \varphi) \\
i G_{n \widetilde{k}}(r) Y_{j m}^{\widetilde{l}}(\theta, \varphi)
\end{array}\right)
$$

where $\sigma_{1}=\left(\begin{array}{ll}0 & 1 \\ 1 & 0\end{array}\right), \sigma_{2}=\left(\begin{array}{cc}0 & -i \\ i & 0\end{array}\right)$ and $\sigma_{3}=\left(\begin{array}{cc}1 & 0 \\ 0 & -1\end{array}\right)$ and are $2 \times 2$ three Pauli matrices while $k(\widetilde{k})$ is related to the total angular momentum quantum numbers for spin symmetry $l$ and p-spin symmetry $\tilde{l}$ as $[18-22]$ :

$$
k=\left\{\begin{array}{l}
-(l+1) \text { if }-(\mathrm{j}+1 / 2),\left(\mathrm{s}_{1 / 2}, p_{3 / 2}, \text { etc }\right), j=l+\frac{1}{2}, \text { aligned } \operatorname{spin}(\mathrm{k}\langle 0) \\
\left.+l \quad \text { if } j=l+\frac{1}{2},\left(\mathrm{p}_{1 / 2}, d_{3 / 2}, \text { etc }\right), j=l-\frac{1}{2}, \text { unaligned } \operatorname{spin}(\mathrm{k}\rangle 0\right)
\end{array}\right.
$$

and

$$
\tilde{k}=\left\{\begin{array}{l}
-\tilde{l} \text { if }-(\mathrm{j}+1 / 2),\left(\mathrm{s}_{1 / 2}, p_{3 / 2}, \text { etc }\right), j=\tilde{l}-\frac{1}{2}, \text { aligned } \operatorname{spin}(\mathrm{k}\langle 0) \\
+(\tilde{l}+1) \text { if } j=\widetilde{l}+\frac{1}{2},\left(\mathrm{p}_{1 / 2}, d_{3 / 2}, \text { etc }\right), j=\widetilde{l}+\frac{1}{2}, \text { unaligned spin }(\mathrm{k}>0)
\end{array}\right.
$$

The radial functions $\left(F_{n k}(r), G_{n k}(r)\right)$ are obtained by solving the following differential equations [1822]:

$$
\left[\frac{d^{2}}{d r^{2}}-\frac{k(k+1)}{r^{2}}-\left(M+E_{n k}-\Delta(r)\left(M-E_{n k}+\Sigma(r)\right)+\frac{\frac{d \Delta(r)}{d r}\left(\frac{d}{d r}+\frac{k}{r}\right)}{M-E_{n k}+\Sigma(r)}\right)\right] F_{n k}(r)=0
$$

and

$$
\left[\frac{d^{2}}{d r^{2}}-\frac{k(k-1)}{r^{2}}\left(M+E_{n k}-\Delta(r)\left(M-E_{n k}+\Sigma(r)\right)+\frac{\frac{d \Sigma(r)}{d r}\left(\frac{d}{d r}+\frac{k}{r}\right)}{M+E_{n k}-\Delta(r)}\right)\right] G_{n \tilde{k}}(r)=0
$$

The exact spin symmetry corresponding $\frac{d \Delta(r)}{d r}=0$, thus the radial function $F_{n k}(r)$ satisfying the following like Schrödinger equation [22]:

$$
\left[\frac{d^{2}}{d r^{2}}-\frac{k(k+1)}{r^{2}}-M^{2}-v_{0} E^{2}(M-E) \frac{e^{-\alpha r}}{r}\right] F_{n k}(r)=0
$$

The relativistic energy $E_{n, k}$ and radial upper wave $F_{n k}(r)$ are given by [22]:

$$
E_{n_{r}, k}=\frac{1}{2}\left\{\left(C_{s}-\alpha v_{0}\right) \pm \sqrt{\left(C_{s}-\alpha v_{0}\right)^{2}-4\left(-M^{2}+C_{s} M+\left(M-C_{s}\right) \alpha v_{0}+\frac{\left(A v_{0}\right)^{2}}{[ \pm 2]\left(L^{2}+1 / 4\right)^{1 / 2} \mp 2 n \pm 1}\right)}\right\}
$$


and

$$
F_{n, k}(r)=N n !\left(\mp 2 E_{n_{r}, k}^{\frac{1}{2}}\right)^{2\left(L^{2}+1 / 4\right)} r^{ \pm\left(L^{2}+1 / 4\right)-\frac{1}{2}} \exp \left( \pm 2 E_{n_{r}, k}^{\frac{1}{2}} r\right) L_{n}^{k}(r)
$$

where $L_{n}^{k}(r)$ stands for the associated Laguerre functions and $L^{2}=k(k+1)$. For, the exact pseudospin symmetry which corresponds $\frac{d \sum(r)}{d r}=0$, the relativistic energy $\widetilde{E}_{n_{r}, k}$ and radial lower wave $G_{n k}(r)$ are given by [22]:

$$
\widetilde{E}_{n_{r}, k}=\frac{1}{2}\left\{C_{p} \pm \sqrt{C_{p}^{2}+4\left(-M^{2}+C_{p} M+A_{p} \alpha v_{0}+\frac{\left(A_{p} v_{0}\right)^{2}}{[ \pm 2]\left(L_{p}{ }^{2}+1 / 4\right)^{1 / 2} \mp 2 n \pm 1}\right)}\right\}
$$

and

$$
G_{n, k}(r) \widetilde{N} \mathrm{n} !\left(\mp 2 \widetilde{E}_{n_{r}, k}^{\frac{1}{2}}\right)^{2\left(L^{2}+1 / 4\right)} r^{ \pm\left(L^{2}+1 / 4\right)-\frac{1}{2}} \exp \left( \pm 2 \widetilde{E}_{n_{r}, k}^{\frac{1}{2}} r\right) L_{n}^{k}(r)
$$

\section{NC relativistic Hamiltonian for modified Yukawa potential:}

\subsection{Formalism of Bopp's shift Method}

In this section I first highlight in brief the basics of the concepts of the quantum noncommutative quantum mechanics in the framework of relativistic Dirac equation for modified Yukawa potential $V_{a o}(\hat{r})$ on based to our works [28-43]:

a) Ordinary Dirac Hamiltonian operator $\hat{H}\left(p_{i}, x_{i}\right)$ replace by NC Dirac Hamiltonian operator $\hat{H}_{n c-y p}\left(\hat{p}_{i}, \hat{x}_{i}\right)$,

b) Ordinary spinor $\Psi(\vec{r})$ replace by new spinor $\Psi(\vec{r})$,

c) Ordinary relativistic energy $\mathrm{E}_{\mathrm{nk}}$ replaces by new relativistic energy $E_{n c-y p}$ and ordinary product replace by new star product*.

Thus, the Dirac equation in ordinary quantum mechanics will change into the modified Dirac equation in extended quantum mechanics for modified Yukawa potential as follows:

$$
\hat{H}_{n c-y p}\left(\hat{p}_{i}, \hat{x}_{i}\right) * \hat{\Psi}(\vec{r})=E_{n c-y p} \hat{\Psi}(\vec{r})
$$

The Bopp's shift method permutes to reduce the above NC equation to simplest form with usual product and translations applied to in space and phase operators:

$$
H_{n c-y p}\left(\hat{p}_{i}, \hat{x}_{i}\right) \psi(\vec{r})=E_{n c-y p} \psi(\vec{r})
$$

Where the new Hamiltonian operator $H_{n c-y p}\left(\hat{p}_{i}, \hat{x}_{i}\right)$ can be expressed in three general varieties: both NC space and NC phase (NC-3D: RSP), only NC space (NC-3D: RS) and only NC phase (NC: 3D-RP) as, respectively:

$$
H_{n c-y p}\left(\hat{p}_{i}, \hat{x}_{i}\right) \equiv H\left(\hat{p}_{i}=p_{i}-\frac{1}{2} \bar{\theta}_{i j} x_{j} ; \hat{x}_{i}=x_{i}-\frac{1}{2} \theta_{i j} p_{j}\right) \text { for } \quad(\mathrm{NC}-3 \mathrm{D}: \mathrm{RSP})
$$




$$
\begin{aligned}
& H_{n c-y p}\left(\hat{p}_{i}, \hat{x}_{i}\right) \equiv H\left(\hat{p}_{i}=p_{i} ; \hat{x}_{i}=x_{i}-\frac{1}{2} \theta_{i j} p_{j}\right) \text { for }(\mathrm{NC}-3 \mathrm{D}: \mathrm{RS}) \\
& H_{n c-y p}\left(\hat{p}_{i}, \hat{x}_{i}\right) \equiv H\left(\hat{p}_{i}=p_{i}-\frac{1}{2} \bar{\theta}_{i j} ; x_{j}, \hat{x}_{i}=x_{i}\right) \text { for } \quad(\mathrm{NC}-3 \mathrm{D}: \mathrm{RP})
\end{aligned}
$$

In recently work, we are interest with the second variety which present by eq. (28) and by the means of the auxiliary two variables $\hat{x}_{i}$ and $\hat{p}_{i}$, the new modified Hamiltonian $H_{n c-y p}\left(\hat{p}_{i}, \hat{x}_{i}\right)$ may be written as follows

$$
H_{n c-y p}\left(\hat{p}_{i}, \hat{x}_{i}\right)=\alpha \hat{\mathrm{P}}+\beta(M+S(\hat{r}))+V_{y p}(\hat{r})
$$

where the modified Yukawa potential $V_{y p}(\hat{r})$ is given by:

$$
V_{y p}(\hat{r})=v_{0} \frac{e^{-\alpha \hat{r}}}{\hat{r}}
$$

The Dirac equation in the presence of above interaction $V_{y p}(\hat{r})$ can be rewritten according Bopp shift method as follows:

$$
(\alpha \mathrm{P}+\beta(M+S(\hat{r}))) \Psi(r, \theta, \varphi)=\left(E_{n c-y p}-V_{y p}(\hat{r})\right) \Psi(r, \theta, \varphi)
$$

The radial functions $\left(F_{n k}(r), G_{n k}(r)\right)$ are obtained by solving two equations:

$$
\begin{aligned}
& {\left[\frac{d}{d r}+\frac{k}{r}\right] F_{n k}(r)=\left[M+E_{n c-y p}-\Delta(\hat{r})\right] G_{n k}(r)} \\
& {\left[\frac{d}{d r}+\frac{k}{r}\right] G_{n k}(r)=\left[M-E_{n c-y p}+\Sigma(\hat{r})\right] G_{n k}(r)}
\end{aligned}
$$

with $\Delta(\hat{r})=V(\hat{r})-S(\hat{r})$ and $\Sigma(\hat{r})=V(\hat{r})+S(\hat{r})$, eliminating $F_{n k}(r)$ and $G_{n k}(r)$ from Eqs. (33) and (34), we can obtain the following two Schrödinger-like differential equations in (NC-3D: RS) symmetries as follows:

$$
\left[\frac{d^{2}}{d r^{2}}-\frac{k(k+1)}{r^{2}}-\left(M+E_{n c-y p}-\Delta(\hat{r})\right)\left(M-E_{n c-y p}+\sum(\hat{r})\right)\right] F_{n k}(r)=0
$$

and

$$
\left[\frac{d^{2}}{d r^{2}}-\frac{k(k-1)}{r^{2}}-\left(M+E_{n c-y p}-\Delta(\hat{r})\right)\left(M-E_{n c-y p}+\Sigma(\hat{r})\right)\right] G_{n k}(r)=0
$$

After straightforward calculations one can obtains the terms $\frac{e^{-\alpha \hat{r}}}{\hat{r}}$ and $\frac{v_{0}}{\hat{r}}$ in (NC-3D: RS) as follows:

$$
\exp (-\delta r)=e^{-\alpha r}+(1+\alpha) \frac{e^{-\alpha r}}{2 r^{3}} \begin{cases}\vec{L} \vec{\Theta} & \text { for the spin symmetric case } \\ \overrightarrow{\overrightarrow{\mathbf{L}}} \vec{\Theta} & \text { for thep-spin symmetric case }\end{cases}
$$

and

$$
\frac{v_{0}}{\hat{r}}=\frac{v_{0}}{r}-\frac{v_{0}}{2 r^{3}} \begin{cases}\vec{L} \vec{\Theta} & \text { for the spin symmetric case } \\ \overrightarrow{\overrightarrow{\mathbf{L}}} \vec{\Theta} & \text { for the } \mathrm{p} \text { - spin symmetric case }\end{cases}
$$


which allow us to writing the modified Yukawa potential $V_{y p}(\hat{r})$ in (NC-3D: RS) as follows:

$$
V_{y p}(\hat{r})=v_{0} \frac{e^{-\alpha r}}{r}+\left\{\begin{array}{l}
\hat{V}_{1 p-y p}\left(r, \Theta, \alpha, v_{0}\right)=v_{0}(1+\alpha) \frac{\vec{L} \vec{\Theta}}{2 r^{3}} e^{-\alpha r} \overrightarrow{\mathbf{L}} \vec{\Theta} \text { for the spin symmetric case } \\
\hat{V}_{2 p-y p}\left(r, \Theta, \alpha, v_{0}\right)=v_{0}(1+\alpha) \frac{\vec{L} \vec{\Theta}}{2 r^{3}} e^{-\alpha r} \widetilde{\overrightarrow{\mathbf{L}}} \vec{\Theta} \text { for thep - spin symmetric case }
\end{array}\right.
$$

It's clearly that, the first term represent the ordinary Yukawa potential while the rest parts $\hat{V}_{1 p-y p}\left(r, \Theta, \alpha, v_{0}\right)$ and $\hat{V}_{2 p-y p}\left(r, \Theta, \alpha, v_{0}\right)$ are produced by the deformation of space, this allows writing the modified Yukawa potential in the NC case as an equation similarly to the usual Dirac equation of the commutative type with a non local potential. Furthermore, using the unit step function (also known as the Heaviside step function or simply the theta function) we can rewrite the modified Yukawa potential to the following form:

$$
V_{y p}(\hat{r})=v_{0} \frac{e^{-\alpha r}}{r}+\theta\left(E_{n c-y p}\right) \hat{V}_{1 p-y p}\left(r, \Theta, \alpha, v_{0}\right)+\theta\left(-E_{n c-y p}\right) \hat{V}_{2 p-y p}\left(r, \Theta, \alpha, v_{0}\right)
$$

where

$$
\theta(x)=\left\{\begin{array}{lll}
1 & \text { for } & x>0 \\
0 & \text { for } & x<0
\end{array}\right.
$$

We generalized the constraint for the pseudospin (p-spin) symmetry $\Delta(r)=V(r)$ and $\Sigma(r)=C_{p s}=$ constants which presented in refs. [18-22] into the new form $\Delta(\hat{r})=V(\hat{r})$ and $\Sigma(\hat{r})=\hat{C}_{p s}=$ constants in (NC-3D: RS) and inserting the potential $V_{y p}(\hat{r})$ in eq. (39) into the two Schrödinger-like differential equations (35) and (36), one obtains:

$$
\begin{aligned}
& {\left[\frac{d^{2}}{d r^{2}}-\frac{k(k+1)}{r^{2}}-\left(M+E_{n c-y p}\right)\left(M-E_{n c-y p}+C_{p s}\right)-v_{0} \frac{e^{-\alpha r}}{r}\left(M-E_{n c-y p}+C_{p s}\right)-v_{0}(1+\alpha) \frac{e^{-\alpha r}}{2 r^{3}}\left(M-E_{n c-y p}+\hat{C}_{p s}\right) \overrightarrow{\mathbf{L}} \vec{\Theta}\right] F_{n k}(r)=0} \\
& {\left[\frac{d^{2}}{d r^{2}}-\frac{k(k-1)}{r^{2}}-\left(M+E_{n c-y p}\right)\left(M-E_{n c-y p}+C_{p s}\right)-v_{0} \frac{e^{-\alpha r}}{r}\left(M-E_{n c-y p}+C_{p s}\right)-v_{0}(1+\alpha) \frac{e^{-\alpha r}}{2 r^{3}}\left(M-E_{n c-y p}+\hat{C}_{p s}\right) \overrightarrow{\mathbf{L}} \vec{\Theta}\right] G_{n k}(r)=0}
\end{aligned}
$$

It's clearly that, the additive two parts $\hat{V}_{1 p-y p}\left(r, \Theta, \alpha, v_{0}\right)$ and $\hat{V}_{2 p-y p}\left(r, \Theta, \alpha, v_{0}\right)$ are proportional with infinitesimal parameter $\Theta$, thus we can considered as a perturbations terms.

4. The exact relativistic spin-orbital Hamiltonian and the corresponding spectrum for modified Yukawa potential in (NC: 3D- RS) symmetries for $n^{\text {th }}$ excited states for one-electron atoms:

4.1 The exact relativistic spin-orbital Hamiltonian for modified Yukawa potential in (NC: 3DRS) symmetries for one-electron atoms:

In this part of the paper, again, the two perturbative terms $\hat{V}_{1 p-y p}\left(r, \Theta, \alpha, v_{0}\right)$ and $\hat{V}_{2 p-y p}\left(r, \Theta, \alpha, v_{0}\right)$ can be rewritten to the equivalent physical form for modified Yukawa potential as follows:

$$
\left\{\begin{array}{l}
\hat{V}_{1 p-y p}\left(r, \Theta, \alpha, v_{0}\right)=\Theta_{0}(1+\alpha) \frac{e^{-\alpha r}}{2 r^{3}} \overrightarrow{\mathbf{L}} \vec{S} \text { for the spin symmetric case } \\
\hat{V}_{2 p-y p}\left(r, \Theta, \alpha, v_{0}\right)=\Theta_{0}(1+\alpha) \frac{e^{-\alpha r}}{2 r^{3}} \overrightarrow{\mathbf{L}} \overrightarrow{\widetilde{S}} \text { for thep-spin symmetric case }
\end{array}\right.
$$


Furthermore, the above perturbative terms $\hat{V}_{1 p-y p}\left(r, \Theta, \alpha, v_{0}\right)$ and $\hat{V}_{2 p-y p}\left(r, \Theta, \alpha, v_{0}\right)$ can be rewritten to the following new equivalent form for (m.a.o.) potential:

$$
\left\{\begin{array}{l}
\hat{V}_{1 p-y p}\left(r, \Theta, \alpha, v_{0}\right)=\frac{1}{2} \Theta v_{0}(1+\alpha) \frac{e^{-\alpha r}}{2 r^{3}}\left(\vec{J}^{2}-\vec{L}^{2}-\vec{S}^{2}\right) \text { for the spin symmetric case } \\
\hat{V}_{2 p-y p}\left(r, \Theta, \alpha, v_{0}\right)=\frac{1}{2} \Theta v_{0}(1+\alpha) \frac{e^{-\alpha r}}{2 r^{3}}\left(\vec{J}^{2}-\vec{L}^{2}-\overrightarrow{\widetilde{S}}^{2}\right) \text { for thep - spin symmetric case }
\end{array}\right.
$$

To the best of our knowledge, we just replace the two spin-orbital coupling $\vec{S} \overleftrightarrow{L}$ and $\widetilde{\overrightarrow{\mathbf{L}}} \overrightarrow{\widetilde{S}}$ by the expression $\frac{1}{2}\left(\vec{J}^{2}-\vec{L}^{2}-\vec{S}^{2}\right)$ and $\frac{1}{2}\left(\vec{J}^{2}-\vec{L}^{2}-\vec{S}^{2}\right)$, in relativistic quantum mechanics. The set $\left(H_{n c-y p}\left(\hat{p}_{i}, \hat{x}_{i}\right), \mathrm{J}^{2}, \mathrm{~L}^{2}, \widetilde{\mathrm{S}}^{2}\right.$ and $\left.J_{z}\right)$ forms a complete of conserved physics quantities and the spinorbit quantum number $k(\tilde{k})$ is related to the quantum numbers for spin symmetry $l$ and p-spin symmetry $\tilde{l}$ as follows [18-21]:

$$
k=\left\{\begin{array}{l}
k_{1} \equiv-(l+1) \quad \text { if }-(\mathrm{j}+1 / 2),\left(\mathrm{s}_{1 / 2}, p_{3 / 2}, \text { etc }\right), j=l+\frac{1}{2}, \text { aligned } \operatorname{spin}(\mathrm{k}\langle 0) \\
\left.k_{2} \equiv+l \quad \text { if }\left(j=l+\frac{1}{2}\right),\left(\mathrm{p}_{1 / 2}, d_{3 / 2}, \text { etc }\right), j=l-\frac{1}{2}, \text { unaligned } \operatorname{spin}(\mathrm{k}\rangle 0\right)
\end{array}\right.
$$

and

$$
\widetilde{k}=\left\{\begin{array}{l}
\tilde{k}_{1} \equiv-\tilde{l} \quad \text { if }-(\mathrm{j}+1 / 2),\left(\mathrm{s}_{1 / 2}, p_{3 / 2}, \text { etc }\right), j=\tilde{l}-\frac{1}{2}, \text { aligned } \operatorname{spin}(\mathrm{k}\langle 0) \\
\tilde{k}_{2} \equiv+(\tilde{l}+1) \quad \text { if }\left(j=\tilde{l}+\frac{1}{2}\right),\left(\mathrm{p}_{1 / 2}, d_{3 / 2}, \text { etc }\right), j=\tilde{l}+\frac{1}{2}, \text { unaligned spin }(\mathrm{k}>0)
\end{array}\right.
$$

With $\tilde{k}(\tilde{k}-1)=\tilde{l}(\tilde{l}+1)$ and $k(k-1)=l(l+1)$, which allows us to form two diagonal $(3 \times 3)$ matrixes $\hat{H}_{s o-y p}\left(k_{1}, k_{2}\right)$ and $\hat{\widetilde{H}}_{s o-y p}\left(\tilde{k}_{1}, \widetilde{k}_{2}\right)$, in (NC: 3D-RS) as:

$\left(\hat{H}_{s o-y p}\right)_{11}\left(k_{1}\right)=k_{1} \Theta v_{0}(1+\alpha) \frac{e^{-\alpha r}}{2 r^{3}} \quad$ if $-(\mathrm{j}+1 / 2),\left(\mathrm{s}_{1 / 2}, p_{3 / 2}, e t c\right), j=l+\frac{1}{2}$, aligned $\operatorname{spin}(\mathrm{k}<0)$

$\left(\hat{H}_{s o-y p}\right)_{22}\left(k_{2}\right)=k_{2} \Theta v_{0}(1+\alpha) \frac{e^{-\alpha r}}{2 r^{3}}$ if $\left(j=l+\frac{1}{2}\right),\left(\mathrm{p}_{1 / 2}, d_{3 / 2}, e t c\right), j=l-\frac{1}{2}$, unaligned $\left.\operatorname{spin}(\mathrm{k}\rangle 0\right)$

$\left(\hat{H}_{s o-y p}\right)_{33}=0$

and

$\left(\hat{H}_{s o-y p}\right)_{11}\left(\widetilde{k}_{1}\right)=\widetilde{k}_{1} \Theta v_{0}(1+\alpha) \frac{e^{-\alpha r}}{2 r^{3}}$ if $-(\mathrm{j}+1 / 2),\left(\mathrm{s}_{1 / 2}, p_{3 / 2}, e t c\right), j=\widetilde{l}-\frac{1}{2}$, aligned $\operatorname{spin}(\mathrm{k}\langle 0)$

$\left(\hat{H}_{s o-y p}\right)_{22}\left(\tilde{k}_{2}\right)=\widetilde{k}_{2} \Theta v_{0}(1+\alpha) \frac{e^{-\alpha r}}{2 r^{3}}$ if $\left(j=\tilde{l}+\frac{1}{2}\right),\left(\mathrm{p}_{1 / 2}, d_{3 / 2}\right.$, etc $), j=\tilde{l}+\frac{1}{2}$, unaligned spin $(\mathrm{k}>0)$

$\left(\hat{H}_{s o-y p}\right)_{33}=0$ 
4.2. The exact relativistic spin-orbital spectrum for modified Yukawa potential symmetries for $n^{\text {th }}$ excited states for one-electron atoms in (NC: 3D- RSP) symmetries:

Our goal in this sub section is to find the modifications to the energy levels $E_{n c-p e r: u}\left(\Theta, k_{1}, E_{n_{r}, k}, \alpha, v_{0}\right)$ and $E_{n c-p e r \cdot d}\left(\Theta, k_{2}, E_{n_{r}, k}, \alpha, v_{0}\right)$ for $\left(-(\mathrm{j}+1 / 2),\left(\mathrm{s}_{1 / 2}, p_{3 / 2}\right.\right.$, etc $), j=l+\frac{1}{2}$, aligned spin $(\mathrm{k}\langle 0)$ and spin-up) and $\left(\left(j=l+\frac{1}{2}\right),\left(\mathrm{p}_{1 / 2}, d_{3 / 2}, e t c\right), j=l-\frac{1}{2}\right.$, unaligned spin $(\mathrm{k}>0)$ and spin down), respectively, at first order of infinitesimal parameter $\Theta$, for excited $n^{\text {th }}$ state, obtained by applying the standard perturbation theory, using Eqs. (22), (44), (45), (46) and (45) as:

$$
\begin{aligned}
& \int \stackrel{+}{\Psi}_{n k}(r, \theta, \varphi)\left[\theta\left(E_{n c-y p}\right) \hat{V}_{1 p-y p}\left(r, \Theta, \alpha, v_{0}\right)+\theta\left(-E_{n c-a o}\right) \hat{V}_{2 p-y p}\left(r, \Theta, \alpha, v_{0}\right)\right] \Psi_{n k}(r, \theta, \varphi) r^{2} d r d \Omega= \\
& =\theta\left(E_{n c-a o}\right) \int F^{*}{ }_{n k}(r) \hat{V}_{1 p-y p}\left(r, \Theta, \alpha, v_{0}\right) F_{n k}(r) d r-\theta\left(E_{n c-a o}\right) \int G_{n \widetilde{k}}^{*}(r) \hat{V}_{2 p-y p}\left(r, \Theta, \alpha, v_{0}\right) G_{n \widetilde{k}}(r) d r
\end{aligned}
$$

The first parts represent the modifications to the energy levels for the spin symmetric cases $E_{n c-p e r: u}\left(\Theta, k_{1}, E_{n_{r}, k}, \alpha, v_{0}\right)$ and $E_{n c-p e r: d}\left(\Theta, k_{2}, E_{n_{r}, k}, \alpha, v_{0}\right)$ while the second part represent the modifications to the energy levels $\left(\widetilde{E}_{n c-p e r: u}\left(\Theta, \widetilde{k}_{1}, \widetilde{E}_{n_{r}, k}, \alpha, v_{0}\right)\right.$ and $\left.\widetilde{E}_{n c-p e r: d}\left(\Theta, \widetilde{k}_{2}, \widetilde{E}_{n_{r}, k}, \alpha, v_{0}\right)\right)$ for the spin spin-symmetry, then we have explicitly:

$$
\begin{aligned}
& E_{n c-p e r: u}\left(\Theta, k_{1}, E_{n k}, \alpha, v_{0}\right) \equiv v_{0} \theta\left(E_{n c-y p}\right) k_{1} \Theta \int^{*} F_{n k}(r)(1+\alpha) \frac{e^{-\alpha r}}{2 r^{3}} F_{n k}(r) d r \\
& E_{n c-p e r: d}\left(\Theta, k_{2}, E_{n k}, \alpha, v_{0}\right) \equiv v_{0} \theta\left(-E_{n c-y p}\right) k_{2} \Theta \int^{*} F_{n k}(r)(1+\alpha) \frac{e^{-\alpha r}}{2 r^{3}} F_{n k}(r) d r
\end{aligned}
$$

Inserting the radial function $F_{n k}(r)$ given by Eq. (22) into the above two Eqs. (51) and (52) to obtain:

$$
\begin{aligned}
& E_{n c-p e r \cdot u}\left(\Theta, k_{1}, E_{n_{r}, k}, \alpha, v_{0}\right) \equiv \\
& \equiv v_{0}(1+\alpha)(\mathrm{n} !)^{2} \Theta k_{1}|N|^{2} \theta\left(E_{n c-y p}\right) \int_{0}^{+\infty} r^{ \pm 2\left(L^{2}+1 / 4\right)-4} \exp \left(\left( \pm 4 E_{n_{r}, k}^{\frac{1}{2}}-\alpha\right) r\right)\left[L_{n}^{k}(r)\right]^{2} d r \\
& E_{n c-p e r \cdot d}\left(\Theta, k_{1}, E_{n_{r}, k}, \alpha, v_{0}\right) \equiv \\
& \equiv v_{0}(1+\alpha)(\mathrm{n} !)^{2} \Theta k_{2}|N|^{2} \theta\left(-E_{n c-y p}\right) \int_{0}^{+\infty} r^{ \pm 2\left(L^{2}+1 / 4\right)-4} \exp \left(\left( \pm 4 E_{n_{r}, k}^{\frac{1}{2}}-\alpha\right) r\right)\left[L_{n}^{k}(r)\right]^{2} d r
\end{aligned}
$$

Now, we introduce the following new factor $T\left(E_{n_{r}, k}, \alpha, L\right)$ as follows:

$$
T\left(E_{n_{r}, k}, \alpha, L\right)=\int_{0}^{+\infty} r^{ \pm 2\left(L^{2}+1 / 4\right)-3-1} \exp \left(\left( \pm 4 E_{n_{r}, k}^{\frac{1}{2}}-\alpha\right) r\right)\left[L_{n}^{k}(r)\right]^{2} d r
$$

we apply the following special integration [72]:

$$
\int_{0}^{+\infty} t^{\alpha-1 .} \exp (-\delta t) L_{m}^{\lambda}(\delta t) L_{n}^{\beta}(\delta t) d t=\frac{\delta^{-\alpha} \Gamma(n-\alpha+\beta+1) \Gamma(m+\lambda+1)}{m ! n ! \Gamma(1-\alpha+\beta) \Gamma(1+\lambda)}{ }_{3} F_{2}(-m, \alpha, \alpha-\beta ;-n+\alpha, \lambda+1 ; 1)
$$

where ${ }_{3} F_{2}(-m, \alpha, \alpha-\beta ;-n+\alpha, \lambda+1 ; 1)$ obtained from the generalized the hypergeometric function ${ }_{p} F_{q}\left(\alpha_{1}, \ldots, \alpha_{p}, \beta_{1}, \ldots, \beta_{q}, z\right)$ for $p=3$ and $q=2$ while $\Gamma(x)$ denote to the usual Gamma function. After straightforward calculations, we can obtain the explicitly results for the factor $T\left(E_{n_{r}, k}, \alpha, L\right)$ : 


$$
\begin{aligned}
T\left(E_{n_{r}, k}, \alpha, L\right) & =\frac{\left(\alpha \mp 4 E_{n_{r}, k}^{\frac{1}{2}}\right)^{\left(\mp 2\left(L^{2}+1 / 4\right)+3\right)} \Gamma\left(n \mp 2\left(L^{2}+1 / 4\right)+k+4\right) \Gamma(n+k+1)}{(n !)^{2} \Gamma\left(4 \mp 2\left(L^{2}+1 / 4\right)+k\right) \Gamma(1+k)} \\
& \times{ }_{3} F_{2}\left(-n, \pm 2\left(L^{2}+1 / 4\right)-3, \pm 2\left(L^{2}+1 / 4\right)-3-k ;-n \pm 2\left(L^{2}+1 / 4\right)-3, k+1 ; 1\right)
\end{aligned}
$$

which allow us to obtain the modifications to the energy levels for $n^{\text {th }}$ excited states:

$$
\begin{aligned}
& E_{n c-p e r u}\left(\Theta, k_{1}, E_{n_{r}, k}, \alpha, v_{0}\right) \equiv v_{0}(1+\alpha)(\mathrm{n} !)^{2} \Theta k_{1}|N|^{2} \theta\left(E_{n c-y p}\right) T\left(E_{n_{r}, k}, \alpha, L\right) \\
& E_{n c-p e r: d}\left(\Theta, k_{1}, E_{n_{r}, k}, \alpha, v_{0}\right) \equiv v_{0}(1+\alpha)(\mathrm{n} !)^{2} \Theta k_{2}|N|^{2} \theta\left(-E_{n c-y p}\right) T\left(E_{n_{r}, k}, \alpha, L\right)
\end{aligned}
$$

\subsection{The exact relativistic magnetic spectrum for modified Yukawa potential for $n^{\text {th }}$ excited states for one-electron atoms in (NC: 3D- RS) symmetries:}

Having obtained the exact modifications to the relativistic energy levels $E_{n c-p e r: u}\left(\Theta, k_{1}, E_{n_{r}, k}, \alpha, v_{0}\right)$ and $E_{n c-p e r d}\left(\Theta, k_{2}, E_{n_{r}, k}, \alpha, v_{0}\right)$ for $n^{\text {th }}$ excited states which produced with relativistic NC spin-orbital Hamiltonian operator, our objective now, in this section, we consider another interested new physically meaningful phenomena, which also can be produce from the perturbative terms of Yukawa potential related to the influence of an external uniform magnetic field, it's sufficient to apply the following three replacements to describing these phenomena:

$$
\begin{aligned}
& \left\{\begin{array}{l}
(1+\alpha) \frac{e^{-\alpha r}}{2 r^{3}} \overrightarrow{\mathbf{L}} \vec{\Theta} \rightarrow \rightarrow \chi(1+\alpha) \frac{e^{-\alpha r}}{2 r^{3}} \vec{B} \vec{L} \text { for the spin symmetric case } \\
(1+\alpha) \frac{e^{-\alpha r}}{2 r^{3}} \overrightarrow{\widetilde{\mathbf{L}}} \vec{\Theta} \rightarrow \rightarrow \chi(1+\alpha) \frac{e^{-\alpha r}}{2 r^{3}} \vec{B} \widetilde{\mathbf{L}} \text { for thep-spin symmetric case }
\end{array}\right. \\
& \Theta \rightarrow \chi B
\end{aligned}
$$

here $\chi$ is infinitesimal real proportional's constants, and we choose the magnetic field $\overleftrightarrow{B}=B \vec{k}$ for simplify the calculations, which allow us to introduce the modified new magnetic Hamiltonian $\hat{H}_{\text {mag-yp }}\left(r, E_{n_{r}, k}, \alpha, v_{0}, \chi\right)$ on the (NC: 3D-RS), as:

$$
\hat{H}_{\text {mag-yp }}\left(r, E_{n_{r}, k}, \alpha, v_{0}\right)=\chi(1+\alpha) \frac{e^{-\alpha r}}{2 r^{3}}\left\{\begin{array}{l}
(\vec{B} \vec{J}-\stackrel{\vec{S}}{\leftrightarrow}) \text { for the spin symmetric case } \\
(\vec{B} \vec{J}-\overrightarrow{\widetilde{S}} \stackrel{\leftrightarrow}{B}) \text { for thep - spin symmetric case }
\end{array}\right.
$$

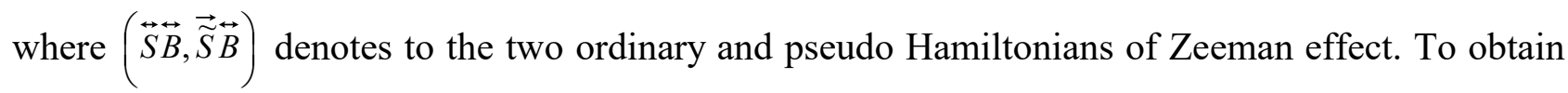
the exact NC magnetic modifications of energy $E_{\text {mag yp }}\left(\chi, m, E_{n_{r}, k}, \alpha, v_{0}\right)$ for modified Yukawa potential under spin-symmetry case which produced automatically from the effect of operator $\hat{H}_{\text {mag-yp }}\left(r, E_{n_{r}, k}, \alpha, v_{0}, \chi\right)$, we make the following two simultaneously replacements:

$$
k_{1} \rightarrow \mathrm{m} \text { and } \Theta \rightarrow \chi
$$


Then, the relativistic magnetic modification of energy $E_{\text {mag-yp }}\left(\chi, m, E_{n_{r}, k}, \alpha, v_{0}\right)$ corresponding ground state on the (NC-3D: RS) symmetries, can be determined from the following relation:

$$
E_{\text {magyp }}\left(\chi, m, E_{n_{r}, k}, \alpha, v_{0}\right)=v_{0}(1+\alpha)(\mathrm{n} !)^{2} \theta\left(E_{n c-y p}\right) \chi m B \Theta|N|^{2} T\left(E_{n_{r}, k}, \alpha, L\right)
$$

The relativistic magnetic modification of energy $\widetilde{E}_{\text {magyp }}\left(\chi, \widetilde{m}, \widetilde{E}_{n_{r}, k}, \alpha, v_{0}\right)$ for pseudospin symmetry can be obtained as:

$$
\widetilde{E}_{\text {magyp }}\left(\chi, \widetilde{m}, \widetilde{E}_{n_{r}, k}, \alpha, v_{0}\right)=v_{0}(1+\alpha)(\mathrm{n} !)^{2} \theta\left(E_{n c-y p}\right) \chi m B \Theta|\widetilde{N}|^{2} T\left(\widetilde{E}_{n_{r}, k}, \alpha, L\right)
$$

where $m$ and $\widetilde{m}$ are represent the angular momentum quantum numbers satisfying the intervals $-l \leq m \leq+l$ and $-\tilde{l} \leq \tilde{m} \leq+\tilde{l}$, which allow us to fixing $(2 l+1)$ and $(2 \tilde{l}+1)$ values.

\section{The main results of exact modified global spectrum for modified Yukawa potential for one-electron atoms under spin-symmetry and p-spin symmetry in (NC: 3D-RS):}

This principal part of the paper is devoted to the presentation of the several results obtained in the previous sections, we resume the $n^{\text {th }}$ excited states eigenenergies $\left(E_{n c-u}\left(\Theta, k_{1}, \chi, n, m, E_{n_{r}, k}, \alpha, v_{0}\right)\right.$, $\left.E_{n c-d}\left(\Theta, k_{2}, \chi, n, m, E_{n_{r}, k}, \alpha, v_{0}\right)\right)$ of modified Dirac equation corresponding for $(-(\mathrm{j}+1 / 2)$, $\left(\mathrm{s}_{1 / 2}, p_{3 / 2}, e t c\right), j=l+\frac{1}{2}$, aligned spin $\mathrm{k}\langle 0$ and spin-down $)$ and $\left(j=l+\frac{1}{2},\left(\mathrm{p}_{1 / 2}, d_{3 / 2}, e t c\right), j=l-\frac{1}{2}\right.$, un aligned spin $k>0$ and spin up), respectively, at first order of parameter $\Theta$, for modified Yukawa potential in (NC: 3D-RS), respectively, on based to the obtained new results (58), (59), (63) and (64), in addition to the original results (21) and (23) of energies in commutative space, we obtain the following original results:

$$
\begin{aligned}
E_{n c-u}\left(\Theta, k_{1}, \chi, n, m, E_{n_{r}, k}, \alpha, v_{0}\right) & =E_{n_{r}, k}+v_{0}(1+\alpha)(\mathrm{n} !)^{2} \Theta k_{1}|N|^{2} \theta\left(E_{n c-y p}\right) T\left(E_{n_{r}, k}, \alpha, L\right) \\
& +v_{0}(1+\alpha)(\mathrm{n} !)^{2} \theta\left(E_{n c-y p}\right) \chi m B \Theta|N|^{2} T\left(E_{n_{r}, k}, \alpha, L\right) \\
E_{n c-d}\left(\Theta, k_{2}, \chi, n, m, E_{n_{r}, k}, \alpha, v_{0}\right) & =E_{n_{r}, k}+\theta v_{0}(1+\alpha)(\mathrm{n} !)^{2} \Theta k_{2}|N|^{2} \theta\left(-E_{n c-y p}\right) T\left(E_{n_{r}, k}, \alpha, L\right) \\
+ & v_{0}(1+\alpha)(\mathrm{n} !)^{2} \theta\left(E_{n c-y p}\right) \chi m B \Theta|N|^{2} T\left(E_{n_{r}, k}, \alpha, L\right)
\end{aligned}
$$

As it is mentioned in ref. [18], in view of exact spin symmetry in commutative space $\left(E_{n k} \rightarrow-E_{n k}, V(r) \rightarrow-V(r), \quad k \rightarrow k+1\right.$ and $F_{n k}(r) \rightarrow G_{n \tilde{k}}(r)$ ), we need to generalize the above translations to the case of $\mathrm{NC}$ three dimensional spaces, then the negative values $E_{n c-u}\left(\Theta, \widetilde{k}_{1}, \chi, m, n, \widetilde{E}_{n_{r}, k}, \alpha, v_{0}\right)$ and $E_{n c-d}\left(\Theta, \widetilde{k}_{2}, \chi, m, n, \widetilde{E}_{n_{r}, k}, \alpha, v_{0}\right)$ are obtained as:

$$
\begin{aligned}
& E_{n c-u}\left(\Theta, k_{1}, \chi, m, n, E_{n_{r}, k}, \alpha, v_{0}\right) \rightarrow E_{n c-u}\left(\Theta, \widetilde{k}_{1}, \chi, m, n, \widetilde{E}_{n_{r}, k}, \alpha, v_{0}\right) \\
& \equiv \widetilde{E}_{n_{r}, k}+v_{0}(1+\alpha)(\mathrm{n} !)^{2} \Theta \widetilde{k_{1}}|\widetilde{N}|^{2} \theta\left(E_{n c-y p}\right) T\left(\widetilde{E}_{n_{r}, k}, \alpha, L\right) \\
& +v_{0}(1+\alpha)(\mathrm{n} !)^{2} \theta\left(E_{n c-y p}\right) \chi m B \Theta|\widetilde{N}|^{2} T\left(\widetilde{E}_{n_{r}, k}, \alpha, L\right) \\
& E_{n c-d}\left(\Theta, k_{2}, \chi, n, m, E_{n_{r}, k}, \alpha, v_{0}\right) \rightarrow E_{n c-d}\left(\Theta, \widetilde{k}_{2}, \chi, m, n, \widetilde{E}_{n_{r}, k}, \alpha, v_{0}\right) \\
& \equiv \widetilde{E}_{n_{r}, k}+\theta v_{0}(1+\alpha)(\mathrm{n} !)^{2} \Theta \widetilde{k}_{2}|\widetilde{N}|^{2} \theta\left(-E_{n c-y p}\right) T\left(\widetilde{E}_{n_{r}, k}, \alpha, L\right) \\
& +v_{0}(1+\alpha)(\mathrm{n} !)^{2} \theta\left(E_{n c-y p}\right) \chi m B \Theta|N|^{2} T\left(E_{n_{r}, k}, \alpha, L\right)
\end{aligned}
$$


It's clearly, that the obtained eigenvalues of energies are real; consequently, the modified quantum Hamiltonian operator $\hat{H}_{n c-y p}\left(\hat{p}_{i}, \hat{x}_{i}\right)$ is Hermitian. Regarding previously obtained results (45) and (62), it is easy to achieve the following equation for Hamiltonian operator $\hat{H}_{n c-y p}\left(\hat{p}_{i}, \hat{x}_{i}\right)$ :

$$
\hat{H}_{n c-a o}\left(\hat{p}_{i}, \hat{x}_{i}\right)=\hat{H}_{c o m-y p}\left(p_{i}, x_{i}\right)+\left\{\begin{array}{l}
\Theta(1+\alpha) \frac{e^{-\alpha r}}{2 r^{3}} \vec{S} \overleftrightarrow{L}+\chi(1+\alpha) \frac{e^{-\alpha r}}{2 r^{3}}(\vec{B} \vec{J}-\overleftrightarrow{\vec{S}} \vec{B}) \text { for the spin symmetric case } \\
\Theta(1+\alpha) \frac{e^{-\alpha r}}{2 r^{3}} \overrightarrow{\widetilde{S}} \overleftrightarrow{L}+\chi(1+\alpha) \frac{e^{-\alpha r}}{2 r^{3}}(\vec{B} \vec{J}-\overrightarrow{\widetilde{S}} \overleftrightarrow{B}) \text { for the p-spin symmetric case }
\end{array}\right.
$$

where $\hat{H}_{c o m-a o}\left(p_{i}, x_{i}\right)$ is the ordinary Hamiltonian operator:

$$
\hat{H}_{c o m-y p}\left(p_{i}, x_{i}\right)=\alpha \mathrm{P}+\beta(M+S(r))+v_{0} \frac{e^{-\alpha r}}{r}
$$

Denote to the ordinary Hamiltonian operator in the commutative space. In this way, one can obtain the complete energy spectra for modified Yukawa potential in (NC: 3D-RS) symmetries. The following accompanying constraint relations:

a) The two quantum numbers $(\tilde{m}, m)$ satisfied the two intervals: $-\tilde{l} \leq \tilde{m} \leq+\tilde{l}$ and $-l \leq m \leq+l$, thus we have $2 \tilde{l}+1$ and $2 l+1$ values for these quantum numbers,

b) We have also two values for p-spin symmetry $j=\tilde{l}+\frac{1}{2}$ and $j=\tilde{l}-\frac{1}{2}$ and two values for spin symmetry $j=l+\frac{1}{2}$ and $j=l-\frac{1}{2}$,

c) For each eigenvalues of $E_{n_{r}, k}$ and $\widetilde{E}_{n_{r}, k}$ we have two physical values.

Allow us to deduce the important original results: every state in usually three dimensional space will be replace by $8(2 \tilde{l}+1)$ and $8(2 l+1)$ sub-states under p-spin symmetry and spin symmetry, which permits to fixing the degenerated states to the $8 \sum_{i=0}^{n-1}(2 l+1) \equiv 8 n^{2}$ values in (NC: $\left.3 \mathrm{D}-\mathrm{RS}\right)$ symmetries. It is easy to see that the obtained originally results reduce to the ordinary results described on quantum mechanics when the noncommutativity of space disappears $(\Theta, \chi) \rightarrow(0,0)$, equations $(65),(66),(67)$ and (68) reduces to (21), (23) and (69) and one recovers the standard textbook results. Finally, one concludes; our obtained results are sufficiently accurate for practical purposes.

\section{The important concluding remarks}

Let us summarize our results as follows:

(i) The solution procedure presented in this paper is based on the both of Bopp's shift method and standard perturbation theory, we investigate the bound state energies of $n^{\text {th }}$ excited states for modified Yukawa potential described on (NC: 3D-RS).

(ii) It is found that the energy eigenvalues depend on the dimensionality of the problem and the new atomic quantum numbers $k(\tilde{k})$ and the two angular momentum quantum numbers $(m, \widetilde{m})$ in addition to the infinitesimal parameters $(\Theta, \chi)$.

(iii) We have also constructing the corresponding NC Hermitian Hamiltonian operator $\hat{H}_{n c-y p}\left(\hat{p}_{i}, \hat{x}_{i}\right)$ which presented by Eq. (68).

The energy eigenvalues are in good agreement with the results previously. Finally, we point out that these exact results (65), (66) and (67) obtained for this new proposed form of the modified Yukawa potential $V_{y p}(\hat{r})$ may have some interesting applications in the study of different quantum mechanical systems, atomic and molecular physics. 


\section{Acknowledgements}

This work was supported with search laboratory of: Physics and Material Chemistry, in Physics department, Sciences faculty, University of M'sila, Algeria.

\section{References}

[1] M.K. Bahar, F. Yasuk, Fermionic particles with position-dependent mass in the presence of inversely quadratic Yukawa potential and tensor interaction, Pramana Journal of Physics. 80(2) (2013) 187-197.

[2] M. Hamzavi, A. A. Rajabi, H. Hassanabadi, Exact pseudospin symmetry solution of the Dirac equation for spatially-dependent mass Coulomb potential including Coulomb-like tensor interaction via asymptotic iteration method, Physics Letters A. 374(42) (2010) 4303-4307.

[3] H. Akcay, C. Tezcan, Exact solutions of the Dirac equation with harmonic oscillator potential including a Coulomb-like tensor interaction, International Journal of Modern Physics C. 20(6) (2010) 931-940.

[4] H. Akcay, Dirac Equation with scalar and vector quadratic potentials and Coulomb-like tensor potential, Physics Letters A. 373(6) (2009) 616-620.

[5] E. Maghsoodi, H. Hassanabadi, S. Zarrinkamar, Exact solutions of the Dirac equation with Poschl-Teller double-ring shaped Coulomb potential via the Nikiforov-Uvarov method, Chinese Physics B. 22(3) (2013) 030302-1-030302-5.

[6] C. Berkdemir, Y. F. Cheng, On the exact solutions of the Dirac equation with a novel angledependent potential, Physica Scripta. 79(3) (2009) 035003-035010.

[7] B.I. Ita, A.I. Ikeuba, Solutions of the Dirac equation with gravitational plus exponential potential, Applied Mathematics. 4 (2013) 1-6.

[8] J. Mawhin, A. Ronveaux, Schrödinger and Dirac equations for the hydrogen atom and Laguerre polynomials, Arch. Hist. Exact Sci. 64 (2010) 429-460.

[9] B. Biswas, S. Debnath, Bound states of the Dirac-Kratzer-Fues problem with spin and pseudospin symmetry via Laplace transform approach, Bulg. J. Phys. 43 (2016) 89-99.

[10] F. Pakdel, A.A. Rajabi, Investigation of the nuclear system using the D-dimensional wave equation, Chinese Journal of Physics. 54 (2016) 385-390.

[11] K.J. Oyewumi, C.O. Akoshile, Bound-state solutions of the Dirac-Rosen-Morse potential with spin and pseudospin symmetry, Eur. Phys. J. A. 45 (2010) 311-318.

[12] H. Hassanabadi, E. Maghsoodi, S. Zarrinkamar, Relativistic symmetry of the Dirac equation and Tietz potential, Eur. Phys. J. Plus. 123 (2012) 31.

[13] M. R. Setare, S. Haidari, Spin symmetry of the Dirac equation with the Yukawa potential, Phys. Scr. 81 (2010) 065201.

[14] A. Soylu, O. Bayrak, I. Boztosun, An approximate solution of the Dirac-Hulthen problem with pseudospin and spin symmetry for any k state, J. Math. Phys. 48(8) (2007) 082302.

[15] C.A. Onate, J.O. Ojonubah, Relativistic and nonrelativistic solutions of the generalized Pöschl-Teller and hyperbolical potentials with some thermodynamic properties, Int. J. Mod. Phys. E. 24 (2015) 1550020.

[16] S.M. Ikhdair, R. Sever, Approximate analytical solutions of the generalized Woods-Saxon potentials including the spin-orbit coupling term and spin symmetry, Cent. Eur. J. Phys. 8 (2010) 665.

[17] S.M. Ikhdair, R. Sever, Approximate eigenvalue and eigenfunctions solutions for the generalized Hulthén potential with any angular momentum, J. Math. Chem. 42(3) (2007) 461471. 
[18] M. Eshghia, S.M. Ikhdair, Relativistic effect of pseudospin symmetry and tensor coupling on the Mie-type potential via Laplace transformation method, Chin. Phys. B. 23(12) (2014) 120304.

[19] S.M. Ikhdair; M. Hamzavi, Approximate relativistic bound state solutions of the Tietz-Hua rotating oscillator for any $\kappa$-state, Few-Body Syst. 53 (2012) 473-486.

[20] M. Hamzavi, A.A. Rajabi, Solution of Dirac equation with Killingbeck potential by using wave function ansatz method under spin symmetry limit, Commun. Theor. Phys. 55(1) (2011) $35-37$.

[21] H. Goudarzi, M. Sohbati, S. Zarrin, Solution of Dirac equation with spin and pseudospin symmetry for an anharmonic oscillator, Journal of Mathematical Physics. 52 (2011) 013506.

[22] M.R. Shojaei et al., Energy levels of spin-1/2 particles with Yukawa interaction, Journal of Modern Physics. 5 (2014) 773-780.

[23] A. Maireche, A study of Schrödinger equation with inverse sextic potential in 2-dimensional non-commutative space, Afr. Rev Phys. 9 (2014) 185-193.

[24] A. Maireche, Spectrum of Schrödinger equation with H.L.C. potential in non-commutative two-dimensional real space, Afr. Rev Phys. 9 (2014) 479-485.

[25] A. Maireche, Deformed bound states for central fraction power potential: non relativistic Schrödinger equation, Afr. Rev Phys. 10 (2015) 97-103.

[26] A. Maireche, Spectrum of hydrogen atom ground state counting quadratic term in Schrödinger equation, Afr. Rev Phys. 10 (2015) 177-183.

[27] A. Maireche, Atomic spectrum for Schrödinger equation with rational spherical type potential in non commutative space and phase, Afr. Rev Phys. 10:0046 (2015) 373-381.

[28] A. Maireche, A complete analytical solution of the Mie-type potentials in non- commutative 3-dimensional spaces and phases symmetries, Afr. Rev Phys. 11:0015 (2016) 111-117.

[29] A. Maireche, A new relativistic study for interactions in one-electron atoms (spin $1 / 2$ particles) with modified Mie-type potential, J. Nano- Electron. Phys. 8(4) (2016) 04027-1 - 04027-9.

[30] A. Maireche, A new nonrelativistic investigation for interactions in one-electron atoms with modified inverse-square potential: noncommutative two and three dimensional space phase solutions at Planck's and nano-scales, J. Nanomed. Res. 4(3) (2016) 00090-00105.

[31] A. Maireche, Investigations on the relativistic interactions in one-electron atoms with modified anharmonic oscillator, J. Nanomed. Res. 4(4) (2016) 00097-00112.

[32] A. Maireche, A new nonrelativistic investigation for interactions in one-electron atoms with modified vibrational-rotational analysis of supersingular plus quadratic potential: extended quantum mechanics, J. Nano- Electron. Phys. 8(4) (2016) 04076-1-04076-9.

[33] A. Maireche, New exact energy eigen-values for (MIQYH) and (MIQHM) central potentials: non-relativistic solutions, Afr. Rev Phys. 11 (2016) 175-185.

[34] A. Maireche, New exact solution of the bound states for the potential family $V(r)=A / r 2-$ $\mathrm{B} / \mathrm{r}+\mathrm{Crk}(\mathrm{k}=0,-1,-2)$ in both noncommutative three dimensional spaces and phases: non relativistic quantum mechanics, International Letters of Chemistry, Physics and Astronomy. 58 (2015) 164-176.

[35] A. Maireche, A new approach to the non relativistic Schrödinger equation for an energydepended potential $V\left(r, E_{n, l}\right)=V_{0}\left(1+\eta E_{n, l}\right) r^{2}$ in both noncommutative three dimensional spaces and phases, International Letters of Chemistry, Physics and Astronomy. 60 (2015) 11-19.

[36] A. Maireche, A new study to the Schrödinger equation for modified potential $\mathrm{V}(\mathrm{r})=\mathrm{ar}^{2}+\mathrm{br}^{-4}+\mathrm{cr}^{-6}$ in nonrelativistic three dimensional real spaces and phases, International Letters of Chemistry, Physics and Astronomy. 61 (2015) 38-48. 
[37] A. Maireche, A new nonrelativistic investigation for the lowest excitations states of interactions in one-electron atoms, Muonic, Hadronic and Rydberg atoms with modified inverse power potential, International Frontier Science Letters. 9 (2016) 33-46.

[38] A. Maireche, Quantum Hamiltonian and spectrum of Schrödinger equation with companied harmonic oscillator potential and it's inverse in three dimensional noncommutative real space and phase, J. Nano- Electron. Phys. 7(4) (2015) 04021-104021-7.

[39] A. Maireche, New relativistic atomic mass spectra of quark ( $\mathrm{u}, \mathrm{d}$ and $\mathrm{s}$ ) for extended modified Cornell potential in Nano and Plank's scales, J. Nano- Electron. Phys. 8(1) (2016) 01020-1 01020-7.

[40] A. Maireche, D. Imane, A new nonrelativistic investigation for spectra of heavy quarkonia with modified Cornell potential: noncommutative three dimensional space and phase space solutions, J. Nano- Electron. Phys. 8(3) (2016) 03024.

[41] A. Maireche, New bound state energies for spherical quantum dots in presence of a confining potential model at Nano and Plank's scales, NanoWorld J. 1(4) (2016) 122-129.

[42] A. Maireche, New exact bound states solutions for (C.F.P.S.) potential in the case of noncommutative three dimensional non relativistic quantum mechanics, Med. J. Model. Simul. 04 (2015) 060-072.

[43] A. Maireche, New quantum atomic spectrum of Schrödinger equation with pseudo harmonic potential in both noncommutative three dimensional spaces and phases, Lat. Am. J. Phys. Educ. 9(1) (2015) 1301.

[44] A. Maireche, Nonrelativistic atomic spectrum for companied harmonic oscillator potential and its inverse in both NC-2D: RSP, International Letters of Chemistry, Physics and Astronomy. 56 (2015) 1-9.

[45] A. Maireche, Deformed quantum energy spectra with mixed harmonic potential for nonrelativistic Schrödinger equation, J. Nano- Electron. Phys. 7(2) (2015) 02003-1 - 02003-6.

[46] A. Maireche, A recent study of quantum atomic spectrum of the lowest excitations for Schrödinger equation with typical rational spherical potential at Planck's and Nanoscales, J. Nano- Electron. Phys. 7(3) (2015) 03047-1 - 03047-7.

[47] A. Maireche, The nonrelativistic ground state energy spectra of potential counting Coulomb and quadratic terms in non-commutative two dimensional real spaces and phases, J. NanoElectron. Phys. 8(1) (2016) 01021-1 - 01021-6.

[48] A. Maireche, New theoretical study of quantum atomic energy spectra for lowest excited states of central (PIHOIQ) potential in noncommutative spaces and phases symmetries at Plan's and Nanoscales, J. Nano- Electron. Phys. 8(2) (2016) 02027-1 - 02027-10.

[49] A. Maireche, A new nonrelativistic atomic energy spectrum of energy dependent potential for heavy quarkouniom in noncommutative spaces and phases symmetries, J. Nano- Electron. Phys. 8(2) (2016) 02046-1 - 02046-6.

[50] A. Maireche, Quantum Schrödinger equation with Octic potential in non-commutative twodimensional complex space, Life Sci. J. 11(6) (2014) 353-359.

[51] A.F. Dossa, G.Y.H. Avossevou, Noncommutative phase space and the two dimensional quantum dipole in background electric and magnetic fields, Journal of Modern Physics. 4 (2013) 1400-1411.

[52] J. Mamat; S. Dulat, H. Mamatabdulla, Landau-like atomic problem on a non-commutative phase space, Int. J. Theor. Phys. 55 (2016) 2913-2918.

[53] Y. Xiao; Z. Long, S. Cai, Klein-Gordon oscillator in noncommutative phase space under a uniform magnetic field, Int. J. Theor. Phys. 50 (2011) 3105-3111. 
[54] A.E.F. Djemaï, H. Smail, On quantum mechanics on noncommutative quantum phase space, Commun. Theor. Phys. 41 (2004) 837.

[55] A. Boumali, H. Hassanabadi, The thermal properties of a two-dimensional Dirac oscillator under an external magnetic field, Eur. Phys. J. Plus. 128 (2013) 124.

[56] S. Dulat, K. Li, The Aharonov-Casher effect for spin-1 particles in non-commutative quantum mechanics, Eur. Phys. J. C. 54 (2008) 333-337.

[57] T. Curtright; D. Fairlie, C.K. Zachos, Features of time independent Wigner functions, Phys. Rev. D. 58 (1998) 025002.

[58] J. Gamboa, M. Loewe, J.C. Rojas, Noncommutative quantum mechanics, Phys. Rev. D. 64 (2001) 067901.

[59] L. Mezincescu, Star operation in quantum mechanics. Available: arXiv: hep-th/0007046v2.

[60] Y. Yuan et al., Spin-1/2 relativistic particle in a magnetic field in NC phase space, Chinese Physics C. 34(5) (2010) 543.

[61] S. Cai, T. Jing, Dirac oscillator in noncommutative phase space, Int. J. Theor. Phys. 49(8) (2010) 1699-1705.

[62] J. Lee, Star products and the Landau problem, Journal of the Korean Physical Society. 47(4) (2005) 571-576.

[63] Y. Zu-Hua et al., DKP oscillator with Spin-0 in three-dimensional noncommutative phase space, Int. J. Theor. Phys. 49 (2010) 644-657.

[64] J. Mamat, S. Dulat, H. Mamatabdulla, Landau-like atomic problem on a non-commutative phase space, Int J Theor Phys; 55 (2016) 2913-2918.

[65] B. Mirza et al., Relativistic oscillators in a noncommutative space and in a magnetic field, Commun. Theor. Phys. 55 (2011) 405-409.

[66] Y. Xiao; Z. Long, S. Cai, Klein-Gordon oscillator in noncommutative phase space under a uniform magnetic field, Int. J. Theor. Phys. 50 (2011) 3105-3111.

[67] A. Al-Jamel, Heavy quarkonia with Cornell potential on noncommutative space, Journal of Theoretical and Applied Physics. 5(1) (2011) 21-24.

[68] H. Hassanabadi, F. Hoseini, S. Zarrinkamar, A generalized interaction in noncommutative space: both relativistic and nonrelativistic fields, Eur. Phys. J. Plus. 130 (2015) 200.

[69] W.S. Chung, Two dimensional non-commutative space and Rydberg atom mode, Int. J. Theor. Phys. 54 (2015) 1840-1849.

[70] P. Polychronakos, Quantum mechanics on the noncommutative plane and sphere, Physics Letters B. 505 (2001) 267.

[71] A.E.F. Djemei, H. Smail, On quantum mechanics on noncommutative quantum phase space, Commun. Theor. Phys. 41 (2004) 837-844.

[72] M. Abramowitz, I.A. Stegun, Handbook of mathematical functions with formulas, graphs and mathematical tables, Dover Publications, New York, 1965. 